\title{
Al tránsito de María (Joseph Conejos Igual, 1745): un ejemplo de reutilización de textos en los villancicos barrocos
}

\section{Al tránsito de María (Joseph Conejos Igual, 1745): an example of re-use of baroque villancico texts}

\author{
Sara Escuer Salcedo \\ Conservatorio Superior de Música de Castilla y León \\ saraescuer@coscyl.com \\ ORCID iD: https://orcid.org/0000-0003-0887-1751
}

\section{RESUMEN}

En este artículo se ofrece un estudio de caso sobre la reutilización de textos en los villancicos barrocos, a partir de un villancico compuesto en 1745 por Joseph Conejos Igual (*1709; †1785), maestro de capilla de la catedral de Jaca (Huesca). El análisis de la obra muestra notables coincidencias con textos literarios de otros villancicos empleados con anterioridad en la catedral de México, en 1685, y en las fiestas por la canonización de San Luis de Gonzaga y San Estanislao de Kotska, en Salamanca, en 1727. El estudio concluye con la edición musical del villancico.

Palabras clave: Joseph Conejos Igual, catedral de Jaca, Sor Juana Inés de la Cruz, villancicos barrocos, reutilización de textos de villancicos.

\section{Abstract}

This article offers a case study on the re-use of baroque villancico texts, based on a villancico composed in 1745 by Joseph Conejos Igual (*1709; †1785), Chapel Master of the Cathedral of Jaca (Huesca). The analysis of the composition shows notable coincidences with literary texts of some villancicos composed some decades before in the Cathedral of 


\section{SARA ESCUER SALCEDO}

Mexico, in 1685, and in the festivities for the canonization of San Luis de Gonzaga and San Estanislao de Kotska, in Salamanca, in 1727. The study concludes with the musical edition of the villancico.

Key Words: Joseph Conejos Igual, Cathedral of Jaca, Sister Juana Inés de la Cruz, Baroque villancicos, re-use of villancico texts.

Escuer Salcedo, S. (2020). Al tránsito de María (Joseph Conejos Igual, 1745): un ejemplo de reutilización de textos en los villancicos barrocos. Cuadernos de Investigación Musical, 10, pp. 39-70.

\section{Al tránsito de MaRía, Villancico a la AsunCión}

Los ocho villancicos que se cantaron en la Santa Iglesia Metropolitana de México, en el día de la Asunción del año 1685, puestos en música por el maestro de capilla, Joseph de Agurto y Loaysa (*1630; †1705) -Joseph de Loaysa y Agurto, en algunas fuentes-, pertenecían a un juego de tres nocturnos escritos por Sor Juana Inés de la Cruz ${ }^{1}$. Sesenta años después, al otro lado del Atlántico, una parte de las letras de cuatro de aquellos villancicos fueron nuevamente musicalizados por el maestro de capilla de la catedral de Jaca, una pequeña ciudad de origen medieval situada al norte de Aragón y a escasos kilómetros de la frontera con Francia.

El análisis del villancico a la Asunción conservado en el archivo musical de la catedral de Jaca (E-J, B/I-573), compuesto en 1745 por el maestro de capilla de la seo altoaragonesa Joseph Conejos Igual ${ }^{2}$, ha permitido confirmar la reutilización de algunos de esos versos de Sor Juana Inés de la Cruz, así como de otros interpretados en la canonización de San Luis de Gonzaga en Salamanca en 1747.

\section{El COMPOSITOR: JOSEPH CONEJOS IGUAL}

Joseph Conejos Igual (*1709; †1785) accedió al cargo de maestro de capilla en Jaca el 3 de julio de 1734 "sin concurso ni examen" gracias a la influencia que ejercieron en el cabildo de la catedral de Jaca "las cartas tan abonatorias de los Maestros de Capilla de la Metropolitana de Zaragoza y otros buenos informes de su suficiencia y calidades que en él concurren"4. Las cartas a las que hacen referencia las actas capitulares del templo jaqués habían sido enviadas desde Zaragoza por los maestros de capilla Joseph Lanuza -de la

\footnotetext{
${ }^{1}$ Los íncipits de los villancicos de Sor Juana Inés de la Cruz analizados en este artículo y musicalizados por Joseph de Agurto y Loaysa están relacionados en: Simón Díaz, J. (1982). Bibliografía de la literatura hispánica. Madrid: Consejo Superior de Investigaciones Científicas-Instituto "Miguel de Cervantes" de Filología Hispánica, vol. XII, pp. 521 y 538.

${ }^{2}$ La información biográfica más completa realizada hasta el momento sobre este maestro de capilla puede consultarse en: Escuer Salcedo, S. (2020). Villancicos y cantadas en la catedral de Jaca (siglo XVIII). Composición, recepción y evolución estilística. Zaragoza: Institución Fernando el Católico, pp. 101-109.

${ }^{3}$ Archivo capitular de la catedral de Jaca, Actas capitulares, (03.07.1734), caja 4, libro 1 (1700-1735), fol. 325v.

${ }^{4}$ Ibidem.
} 
catedral metropolitana- y Luis Serra - del templo de El Pilar-. Joseph Lanuza, en la primera de las dos misivas que envió al cabildo jaqués", no dudó en "asegurar a Vuestra Señoría de la conocida habilidad del pretendiente [...] pues corresponde a la grandeza de la Escuela que ha tenido pasando la composición por todos sus grados con el Maestro D. Luis Serra que lo es del Santo Templo del Pilar]". Por su parte, Luis Serra indicaba haber explicado a su discípulo Joseph Conejos [Igual] "los medios para que saliese aprovechado en la facultad, lo que, gracias a Dios, (y sin vanidad) se ha logrado, como lo publican las obras que de él se han cantado en esta Santa Iglesia"”.

Sin duda, las relaciones familiares y profesionales de J. Conejos Igual favorecieron la recepción en Jaca de obras de compositores vinculados a sedes musicales lejanas, entre las que destacan las catedrales de Valencia y Segorbe -de donde llegaron obras de Antonio T. Ortells o Joseph Conejos Ortells ${ }^{8}{ }^{8}$, así como Zaragoza, ciudad musicalmente muy vinculada a Jaca, ya que varios miembros de las capillas de música de la catedral metropolitana y el templo de El Pilar mantuvieron una estrecha relación profesional con algunos maestros que ejercieron en Jaca durante la primera mitad del siglo XVIII ${ }^{9}$.

En 1749, quince años después su llegada a Jaca, el cabildo catedralicio dejó constancia en las actas capitulares de que "el Maestro de Capilla se había acomodado en otra parte, y que estaba vacante el empleo"10. Aunque la fuente no indica el destino posterior del compositor, recientes investigaciones me permitieron completar el ciclo vital del músico, que presentó su renuncia como maestro de capilla de la catedral jaquesa para ocupar el mismo puesto en la colegiata de Alquézar, villa medieval cercana a Jaca, en la que permaneció hasta su muerte, en $1785^{11}$.

\footnotetext{
${ }^{5}$ La primera de ellas, fechada el 29 de junio de 1734, es una carta de aval para el joven pretendiente; la segunda, enviada el 7 de julio del mismo año, es una carta de agradecimiento al cabildo por el nombramiento del nuevo maestro de capilla. Archivo capitular de la catedral de Jaca, Correspondencia (29.06.1734 y 07.07.1734), caja 33. La transcripción completa de estas dos cartas puede consultarse en: Escuer Salcedo, S. (2020). Villancicos y cantadas en la catedral de Jaca... (op. cit.), pp. 204-205.

6 Archivo capitular de la catedral de Jaca. Correspondencia (29.06.1734), caja 33. [Carta de Joseph Lanuza, maestro de capilla de La Seo de Zaragoza, al cabildo de la catedral de Jaca].

${ }^{7}$ Ibidem, (30.06.1734) [Carta de Luis Serra, maestro de capilla de El Pilar de Zaragoza, al cabildo de la catedral de Jaca].

${ }^{8}$ Fruto de mi investigación presentada como Tesis Doctoral (2017) pude establecer el vínculo familiar entre Joseph Conejos Igual y su tío Joseph Conejos Ortells, maestro de capilla de la catedral de Segorbe. Este último, a su vez, era sobrino de Antonio T. Ortells, maestro de capilla de la catedral de Valencia. Los tres músicos habían nacido en la localidad de Rubielos de Mora (Teruel), en cuya colegiata pudieron haber iniciado su formación musical.

${ }^{9}$ Entre los músicos establecidos en Zaragoza y vinculados profesionalmente con maestros de capilla de la catedral de Jaca destacan el arpista Gerónimo Deza y un Tiple de El Pilar (de nombre desconocido), que fueron invitados por el cabildo jaqués en 1731, a propuesta del maestro de capilla Francisco Viñas, quien probablemente también conociese a Luis Serra. Para una mayor profundización en este tema, véase: Escuer Salcedo, S. (2018). Francisco Viñas y la nueva concepción de la música en la catedral de Jaca (1722-1731). Anuario Musical, LXXIII, pp. 163-164.

${ }_{10}$ Archivo capitular de la catedral de Jaca. Actas capitulares (18.10.1749), caja 4, libro 2 (1700-1735), fol. 144v.

11 Para la consulta de los resultados obtenidos durante mis investigaciones en la colegiata de Alquézar, en relación al maestro de capilla Joseph Conejos Igual, remito al lector a la monografía en la que han sido recogidos: Escuer Salcedo, S. (2020). Villancicos y cantadas en la catedral de Jaca... (op. cit.). A partir del índice geográfico incluido al final de dicha publicación (p. 280) se facilita el acceso a la información obtenida en las investigaciones llevadas a cabo en el archivo de la citada colegiata.
} 


\section{SARA ESCUER SALCEDO}

En el archivo-biblioteca de la colegiata de Alquézar se localizan fuentes documentales que permiten ubicar a J. Conejos Igual al frente de la capilla de música durante más de 45 años, sin embargo, no se conserva ninguna fuente musical de este compositor ni de ningún otro a lo largo de todo el siglo XVIII.

\section{DESCRIPCIÓN DE LA FUENTE MUSICAL}

El villancico $A l$ tránsito de María es una de las 150 obras en lengua romance conservadas en el archivo jaqués y firmadas por el compositor rubielano (a las que se suman 64 obras de diversos géneros en latín), lo que las convierte en las únicas composiciones conocidas de Joseph Conejos Igual.

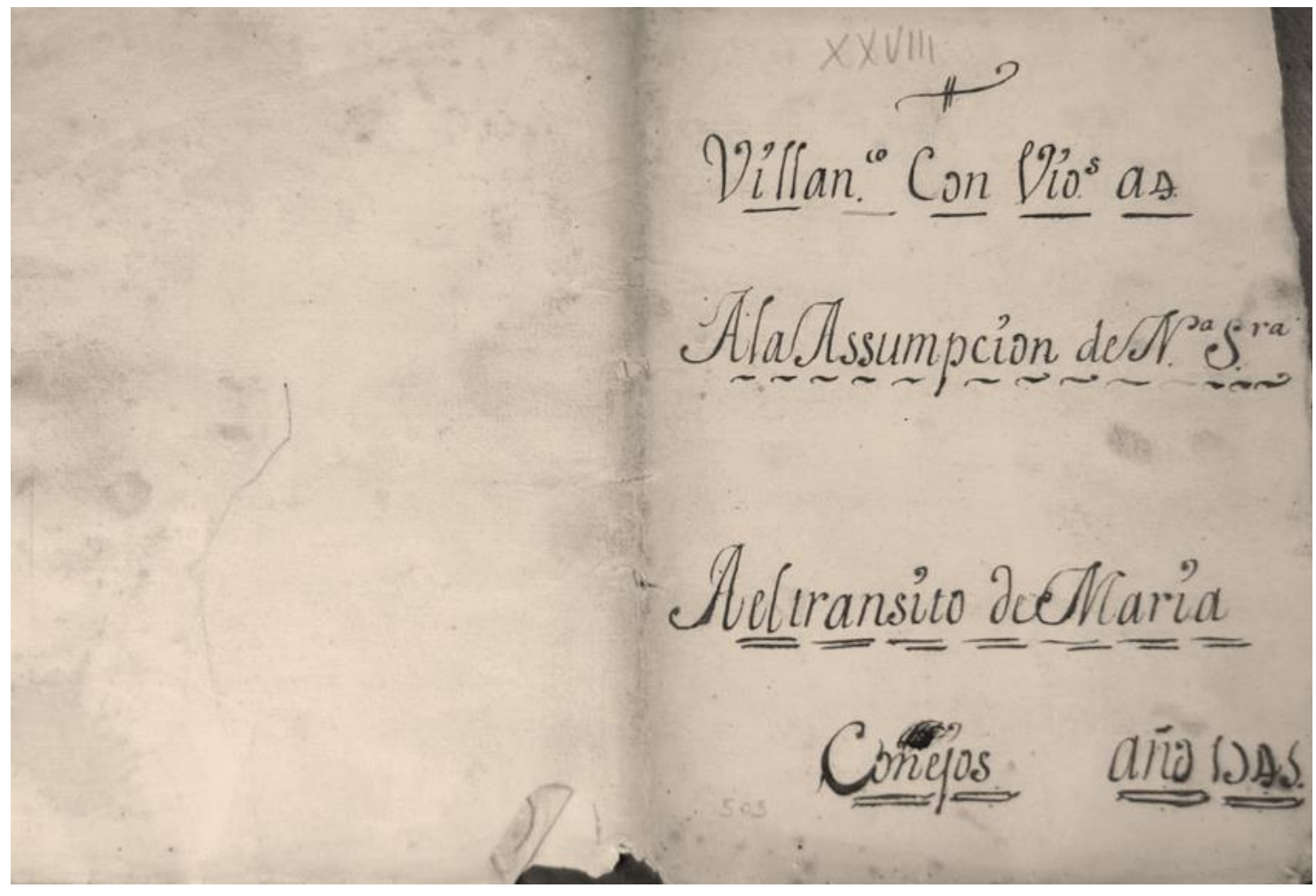

Fig. 1: Portadilla de la obra (Tenor, fol. 1v.).

Se trata de un villancico a 4 voces (dos tiples, alto y tenor) con violín y acompañamiento (bajón obligado y bajo cifrado). La fuente musical consta de siete partes -cuatro vocales y tres instrumentales- anotadas en pliegos por el propio compositor. (Figs. 2 y 3). 
AL TRÁNSITO DE MARÍA (JOSEPH CONEJOS IGUAL, 1745):

UN EJEMPLO DE REUTILIZACIÓN DE TEXTOS EN LOS VILLANCICOS BARROCOS

\begin{tabular}{|c|c|c|c|c|}
\hline Localización: & \multicolumn{2}{|c|}{ Archivo Musical de la Catedral de Jaca } & Signatura: & $\boldsymbol{E}-J, \mathrm{~B} / \mathrm{I}-573$ \\
\hline Compositor: & \multicolumn{2}{|c|}{ Joseph Conejos Igual (*1709; †1785) } & Año: & 1745 \\
\hline \multicolumn{5}{|c|}{ Título diplomático: } \\
\hline $\begin{array}{l}\text { Género y } \\
\text { advocación }\end{array}$ & $\begin{array}{l}\text { Villancico a la } \\
\text { Asunción }\end{array}$ & $\begin{array}{l}\text { Medidas } \\
\text { (alto x ancho): }\end{array}$ & x $31,2 \mathrm{~cm}$ & $\begin{array}{l}\text { Manuscrito } \\
\text { autógrafo }\end{array}$ \\
\hline $\mathbf{n}^{\circ}$ partes: 7 & \multicolumn{2}{|c|}{ Coro: S 1, 2, A, T; vl; bc: bajón, bc: b.fig } & \multicolumn{2}{|c|}{$\mathbf{n}^{\mathbf{o}}$ fols.: $\quad 2,1,1,1 ; 1 ; 1,1 \mathrm{f}$. } \\
\hline
\end{tabular}

Fig. 2: Descripción de la fuente.

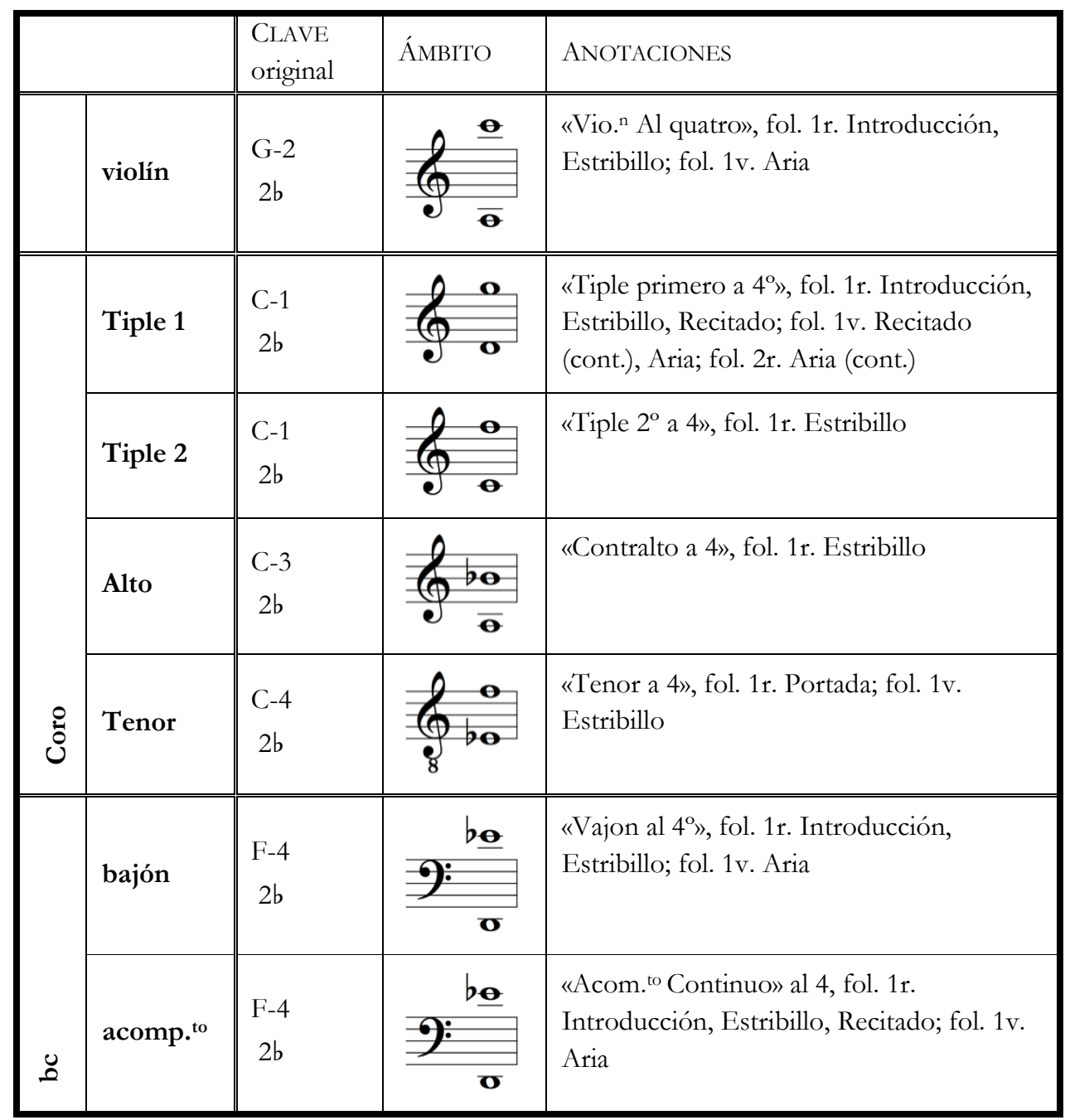

Fig. 3: Descripción de las partes vocales e instrumentales. 


\section{SARA ESCUER SALCEDO}

Si bien la fuente musical indica el término 'villancico', la obra presenta características propias de las cantadas -vocablo utilizado por los compositores españoles para denominar una tipología dentro del género del villancico, surgida por influencia de la cantata italiana y formada por alternancia de recitados y arias-.

En el caso de las composiciones de los maestros de la seo altoaragonesa -al igual que sucedió en otras sedes musicales hispánicas-, a partir de la llegada del maestro de capilla Francisco Viñas en 1722 -responsable de uno de los cambios estilísticos más notables acontecidos en la música en el templo jaqués- y durante todo el siglo XVIII, los compositores continuaron empleando el 'villancico', en convivencia con la nueva tipología, 'cantada', que pronto se expandió por la península. De este modo, indistintamente de la estructura interna de las composiciones en lengua romance y de la presencia de secciones heredadas del siglo anterior (introducción, estribillo y coplas), de nuevas secciones importadas de Italia (recitados y arias) o de la combinación de unas y otras, las fuentes musicales del archivo jaqués ponen de manifiesto el criterio empleado por los compositores para diferenciar villancicos y cantadas en función de los recursos vocales empleados (coro vs. solo o dúo), y no de la estructura formal de las obras.

El villancico Al tránsito de María, de Joseph Conejos Igual, está formado por introducción, estribillo, recitado y aria (Fig. 4), siendo un claro ejemplo de la convivencia entre la tradición y las innovaciones surgidas en la península a comienzos del siglo, coexistencia que caracterizó la música en los templos hispánicos durante el Setecientos.

\begin{tabular}{|l|l|l|}
\hline Sección & Incipit literario & Intervinientes \\
\hline INTRODUCCIÓN & Al tránsito de María & S 1; vl; bajón, acomp. ${ }^{\text {to }}$ \\
\hline ESTRIBILLO & Triunfante señora & S 1, 2, A, T; vl; bajón, acomp..$^{\text {to }}$ \\
\hline RECITADO & Dime enigma sagrado & $\mathrm{S} 1 ;$ acomp. ${ }^{\text {to }}$ \\
\hline ARIA & A la que triunfante & $\mathrm{S} 1 ;$ vl; bajón, acomp. ${ }^{\text {to }}$ \\
\hline
\end{tabular}

Fig. 4: Descripción de las secciones de la obra.

\section{UN EJEMPLO DE REUTILIZACIÓN DE VILLANCICOS}

El análisis literario de la obra muestra que J. Conejos Igual utilizó textos de cuatro composiciones anteriores, procedentes de dos fuentes distintas. Por una parte, varias secciones del villancico presentan coincidencias con tres de los villancicos que se cantaron en 1685 en la Santa Iglesia Metropolitana de México, con motivo de la festividad de la Asunción de María. Estos textos fueron recogidos en la antología Inundación Castálida, de Sor Juana Inés de la Cruz, publicada cuatro años más tarde (Fig. 5). Por otra parte, el recitado reutiliza de forma casi idéntica el empleado en uno de los villancicos que se cantaron en Salamanca durante las fiestas por la canonización de San Luis de Gonzaga y San Estanislao de Kotska, en julio de 1727 (Fig. 6). 


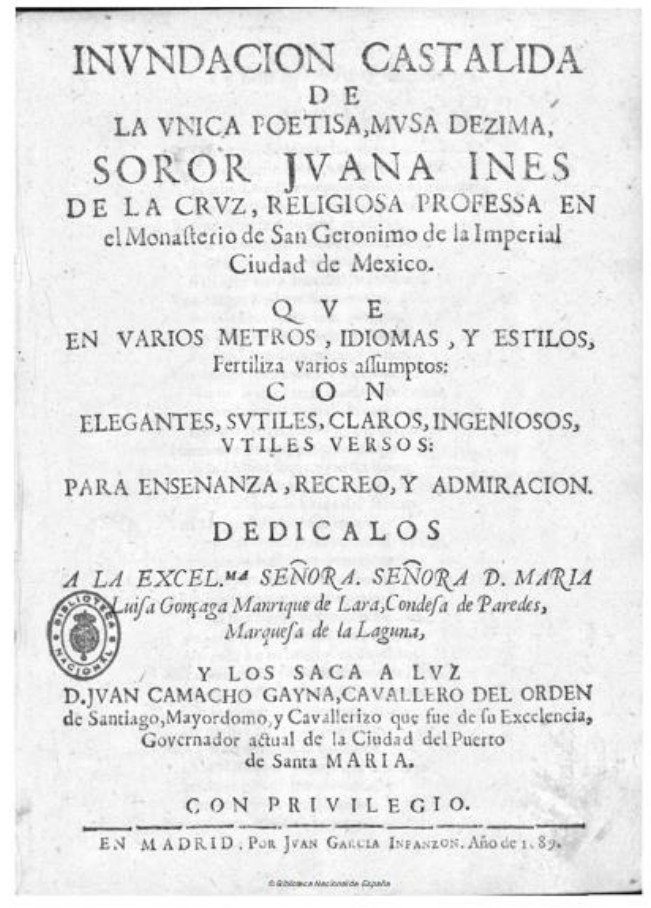

Fig 5: Portada de Inundación castálida de Sor Juana Inés de la Cruz (1689) (Cruz, 1689).

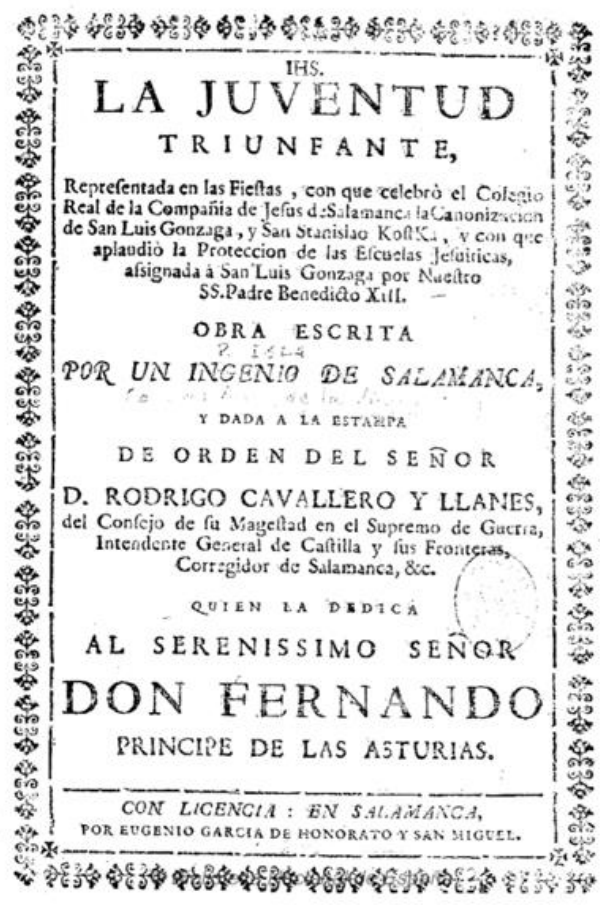

Fig 6: Portada de La juventud triunfante [1727] (Cruz, [1727])

A continuación, se ofrece un análisis comparativo de los diferentes textos reutilizados en cada una de las secciones del villancico de 1745.

La introducción del villancico compuesto en Jaca coincide con las coplas $1^{\mathrm{a}}$ y $2^{\mathrm{a}}$ del villancico I del primer nocturno cantado en México en 1685 (Fig. 7): 


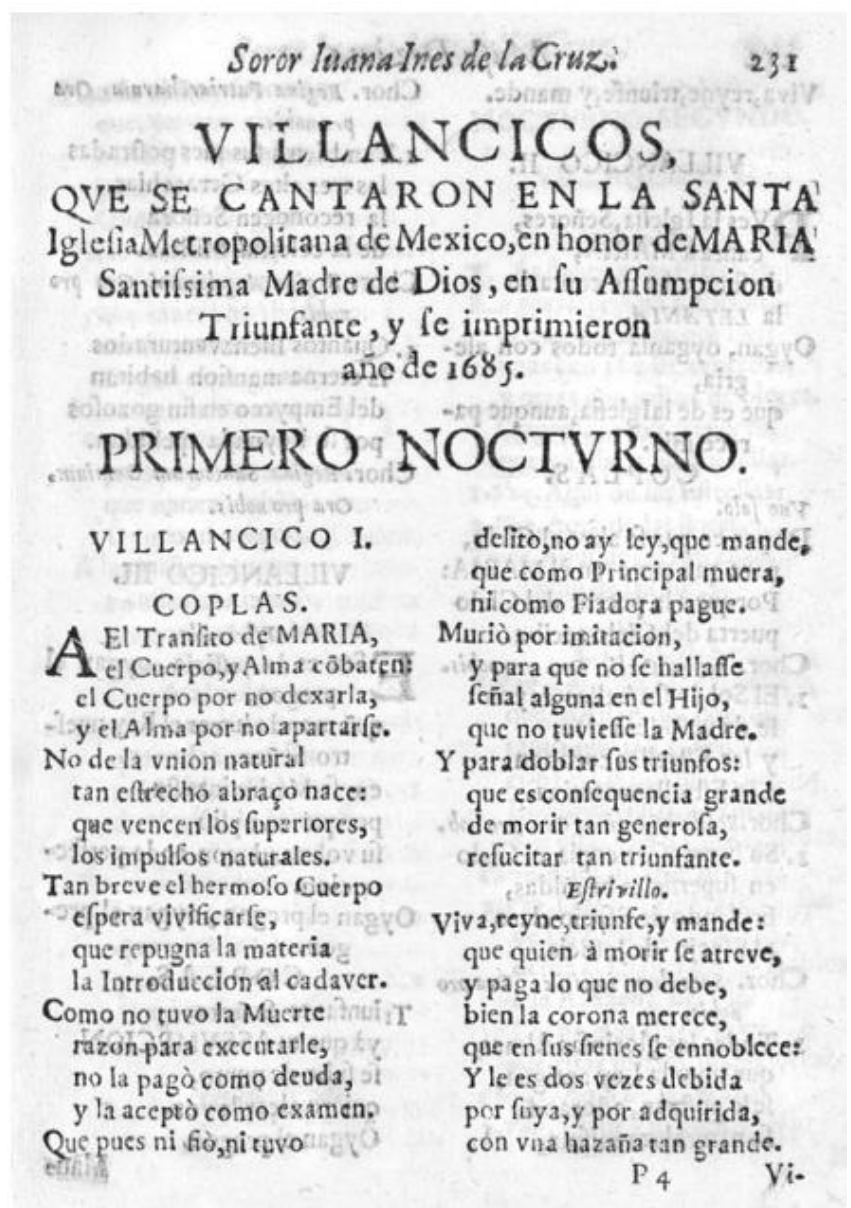

Fig. 7: Villancicos que se cantaron el día de la Asunción de 1685. en la catedral de México. Inundación castálida, p. 231.

\section{Joseph ConeJos IGUAL \\ Catedral de Jaca, 1745}

\section{- Villancico a la Asunción -}

\section{INTRODUCCIÓN}

$1^{a}$. Al tránsito de María

el cuerpo y alma combaten,

el cuerpo por no dejarla

y el alma por no apartarse.

$2^{a}$. No de la unión natural tan estrecho abrazo nace, que vencen los superiores los impulsos naturales.
Sor Juana Inés DE LA CRUZ

Iglesia Metropolitana de México, 1685

\section{- Día de la Asunción - \\ Primer nocturno. Villancico I}

\section{COPLAS [7]}

[1ª A el tránsito de María el cuerpo, y alma combaten; el cuerpo por no dejarla, y el alma por no apartarse.

$\left[2^{a}\right]$ No de la unión natural tan estrecho abrazo nace, que vencen los superiores, los impulsos naturales. 
El estribillo, por su parte, emplea los textos de las cuatro primeras coplas del villancico III, del mismo nocturno.
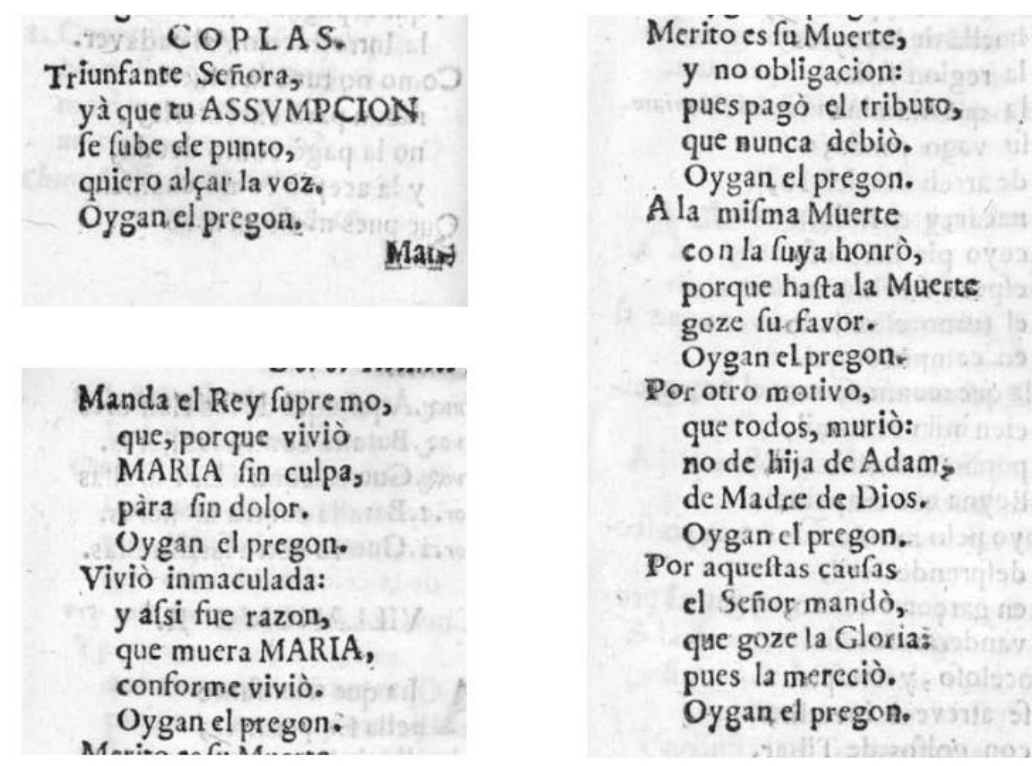

Fig. 8: Villancicos que se cantaron el día de la Asunción de 1685 en la catedral de México. Inundación castálida, pp. 232 y 233.

\section{Joseph CONEJOS IGUAL \\ Catedral de Jaca, 1745}

- Villancico a la Asunción -

\section{ESTRIBILLO}

Triunfante señora,

ya que tu asunción

se sube de punto

quiero alzar la voz.

Manda el rey supremo

que porque vivió

María sin culpa

muera sin dolor.

Vivió inmaculada

y así fue razón

que muera María

conforme vivió.

Mérito es su muerte

y no obligación,

pues pagó el tributo

que nunca debió.
Sor Juana Inés DE LA CRUZ

Iglesia Metropolitana de México, 1685

- Día de la Asunción -

Primer nocturno. Villancico III

\section{COPLAS [7]}

$\left[1^{a}\right]$ Triunfante señora,

ya que tu asunción

se sube de punto

quiero alzar la voz. / Oigan el pregón.

[2] Manda el rey supremo,

que, porque vivió

María sin culpa,

para sin dolor. / Oigan el pregón.

[3] Vivió inmaculada,

$\mathrm{V}$ así fue razón,

que muera María,

conforme vivió. / Oigan el pregón.

$\left[4^{x}\right]$ Mérito es su muerte, y no obligación, pues pagó el tributo, que nunca debió. / Oigan el pregón. 


\section{SARA ESCUER SALCEDO}

El recitado, compuesto para Tiple, utiliza un texto procedente de las Letrillas a san Luis de Gonzaga (Fig. 9) que se interpretaron en Salamanca el 6 de julio de 1727, en uno de los múltiples actos celebrados con motivo de las fiestas por la canonización de san Luis de Gonzaga y San Estanislao de Kostka, y en los que intervino la capilla de música de la catedral salmantina ${ }^{12}$.

Hizo su cumplido con las gracias de la Poesía y de la Música, y pasó a la calle de la Rúa, en cuya entrada la [sic] salió al encuentro el Altar de la Platería, armado de punta en blanco con el más cumplido y luciente arnés que han visto las armerías [...] La Música tocaría entre tanto, y cantaría, como es de creer de su buen natural. Pero las almas de los circunstantes dejaron los sentidos, y se andaban paseando por los cuatro Cuerpos del Altar, como si fueran suyos. Y porque en todos los Altares hubo estos gustos embarazos, y de lo bueno de las Letras Poéticas apenas hubo quien pudiese ser Juez, por no ser oidor, se trasladan aquí para los curiosos. Ojalá se pudiera trasladar también la destreza y melodía de las voces, en que se esmeró la Música de la Catedral. Las Letras son estas (Losada, [1727], p. 67).

LETRILLA A S, LUIS GONZAGA, SOBRE LAVOZ, con que le llamò la Virgen a la Compañia.

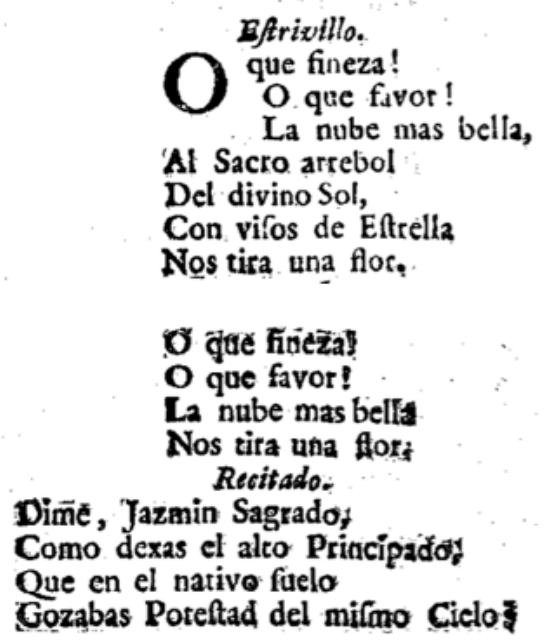

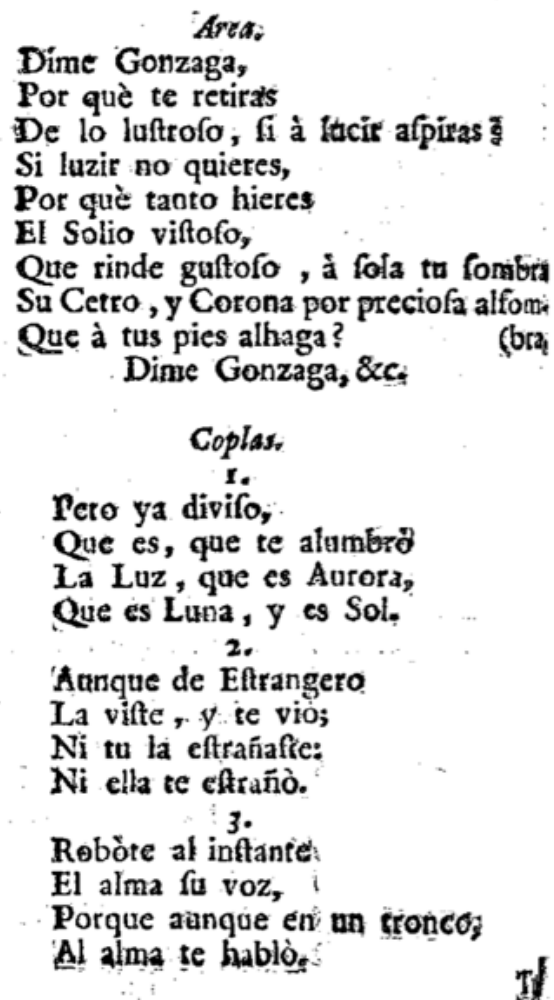

Fig 9: Letrilla a San Luis de Gonzaga. La juventud triunfante, pp. 67 y 68.

\footnotetext{
${ }^{12}$ Las celebraciones se prolongaron durante más de diez días, desde la víspera del día 6 hasta el 17 de julio y fueron narradas por el jesuita Luis de Losada en: La juventud triunfante, Representada en las Fiestas, con que celebró el Colegio Real de la Compañia de Jesús de Salamanca la Canonización de San Luis de Gonzaga, y San Stanislao Kostka, y con que aplaudió la Proteccion de las Escuelas Jesuisticas asignada a San Luis Gonzaga por Muestro SS. Padre Benedicto XIII. Salamanca, Eugenio Garcia de Honorato y San Miguel, [1727].
} 
La primera parte del recitado del villancico de J. Conejos Igual muestra de forma casi idéntica el recitado incluido en la fuente salmantina, con una ligera variación en el primer verso, en el que sustituye 'jazmín' por 'enigma'. La segunda parte del recitado compuesto a la Asunción parece inspirada en los elementos mencionados en la primera copla a san Luis de Gonzaga -diviso, luz, aurora, luna, sol-, y adaptada para la Asunción de la Virgen.

\section{Joseph CONEJOS IGUAL \\ Catedral de Jaca, 1745}

\section{- Villancico a la Asunción -}

\section{RECITADO}

Dime, enigma sagrado,

[j] cómo dejas el alto principado

que en el nativo suelo

gozabas potestad del mismo cielo[?]

Ya en tu asunción diviso

que aspiras al celeste paraíso

porque el cielo te adora,

luz del sol, de la luna y de la aurora.
Luis DE LOSADA

Salamanca, 1727

La juventud triunfante

\section{- Letrilla a san Luis Gonzaga -}

\section{RECITADO}

Dime, jazmin sagrado,

[j]cómo dejas el alto principado

que en el nativo suelo

gozabas potestad del mismo cielo[?]

Finalmente, el aria compuesta por Joseph Conejos Igual presenta coincidencias con los villancicos puestos en música por Sor Juana Inés en 1685. En este caso, corresponden al segundo villancico del segundo nocturno.
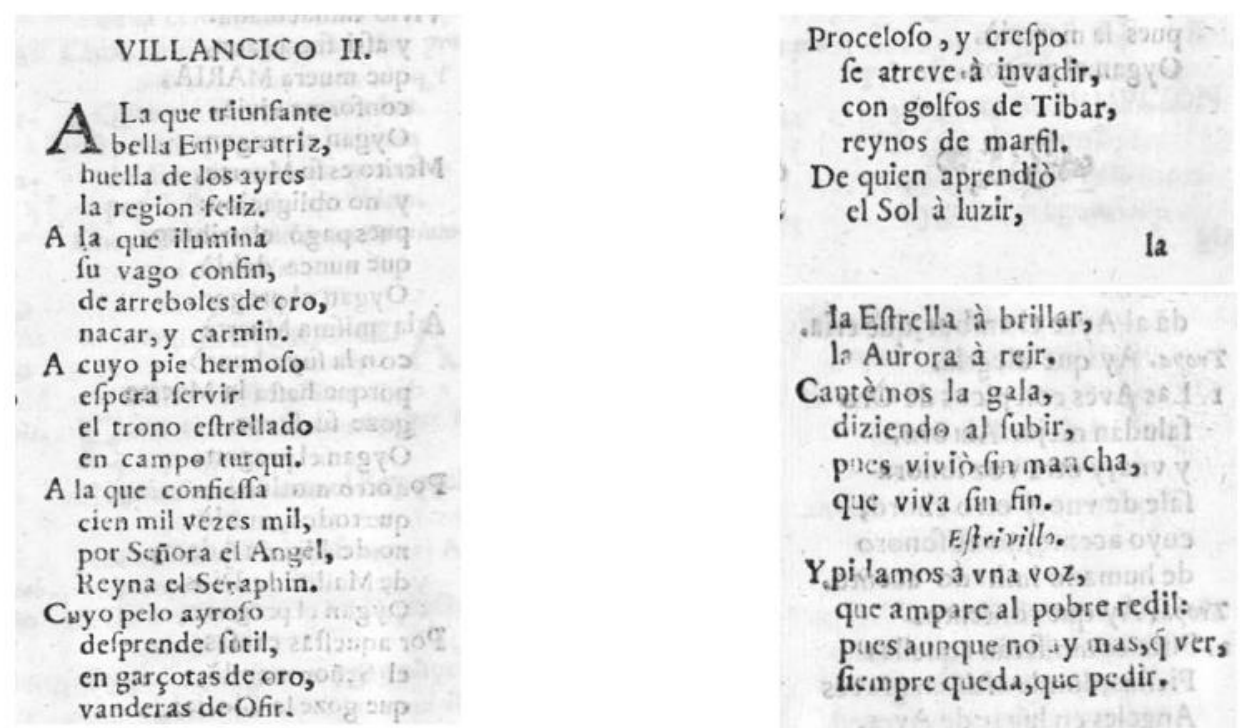

Fig. 10: Villancicos que se cantaron el día de la Asunción de 1685 en la catedral de México. Inundación castálida, pp. 234 y 235. 
Joseph ConEJos IGUAL

Catedral de Jaca, 1745

- Villancico a la Asunción -

ARIA

A la que triunfante, bella, emperatriz, huella de los aires, la región feliz.

Cantemos la gala diciendo al subir pues vivió sin mancha que viva $\sin$ fin.
Sor Juana Inés DE LA CRUZ

Iglesia Metropolitana de México, 1685

- Día de la Asunción Nocturno segundo. Villancico II

[COPLAS]

[1 $\left.1^{2}\right]$ A la que triunfante bella emperatriz, huella de los aires la región feliz.

[COPLAS $2^{\mathrm{a}}$ a $7^{\mathrm{a}}$ ]

[8²] Cantemos la gala, diciendo al subir, pues vivió sin mancha, que viva $\sin$ fin.

\section{[ESTRIBILLO]}

$Y$ pidamos a una voz, que ampare al pobre redil, pues, aunque no hay más que ver, siempre queda que pedir.

\section{RECUPERACIÓN MUSICAL}

\subsection{NOTAS CRÍTICAS}

\section{INTRODUCCIÓN:}

En el manuscrito aparece la indicación de compás de 3/8, pero la figuración utilizada corresponde a un compás de $3 / 4$, por lo que se transcribe en compás $3 / 4$.

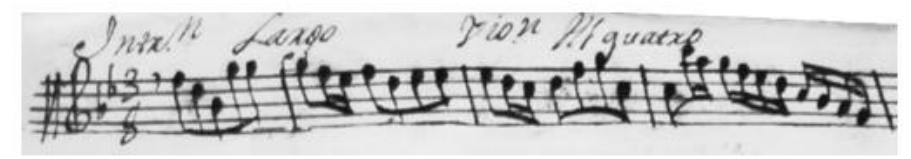

Fig. 11: Violín; introducción (cc. 1-4). 
AL TRÁNSITO DE MARÍA (JOSEPH CONEJOS IGUAL, 1745):

UN EJEMPLO DE REUTILIZACIÓN DE TEXTOS EN LOS VILLANCICOS BARROCOS

c. 15, Tiple 1. Aparece un silencio dentro de un melisma sobre la palabra «combaten». Se mantiene.

c. 16, bajón. La nota Mi está rectificada y la corrección está reforzada con la indicación «fa» en letra sobre ella. Sin embargo, por paralelismo con el acompañamiento, se transcribe Mi.

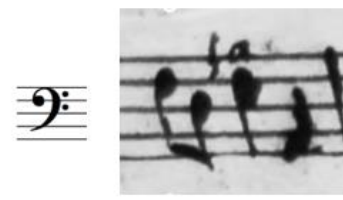

Fig. 12: bajón (c. 16)

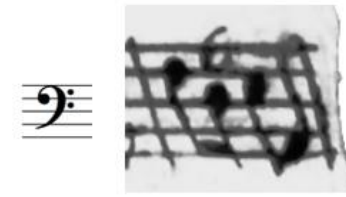

Fig. 13: acompañamiento (c. 16).

cc. 22 y 31, violín. La línea melódica de los cc. 18-26 se repite de forma idéntica y está escrita dos veces (cc. 27-34). Sin embargo, en el c. 22 y en su posterior repetición en el c. 31 las semicorcheas aparecen agrupadas de forma diferente. En la transcripción se mantienen las dos agrupaciones diferentes, como en el manuscrito.

\section{ESTRIBILLO:}

c. 16, violín. Entre los cc. 15 y 16 del manuscrito falta un compás. Al no encontrar similitud en las partes de violín en posteriores apariciones del mismo motivo melódico vocal, se anota un compás, a modo de sugerencia y entre corchetes, correspondiente al c. 16 de la transcripción.

c. 39, Tiple 1. Aparece un silencio expresivo separando la palabra «A-sunción».

c. 87 , violín. En el manuscrito falta un compás. Se sugiere, en su lugar y entre corchetes, el mismo grupo de cuatro corcheas que aparecerán en los cc. 90 y 93, donde el Tiple 2 y el Tiple 1, respectivamente, repiten el mismo elemento melódico del c. 86, tras comprobar que no ocasiona ningún conflicto armónico.

ARIA:

c. 6, violín. En el manuscrito la nota Mi no lleva alteración. La frase de los cc. 1-7 se repite (violín, bajón y acompañamiento) en los cc. 12-18. Teniendo en cuenta esto, se transcribe el c. 6 igual que el c. 17, añadiendo un bemol sobre la nota Mi.

c. 26, Tiple 1. En la nota Mi está registrado un becuadro -actualmente innecesario, puesto que han transcurrido nueve compases desde la aparición de un Mi bemol-, como advertencia para el intérprete, ya que se forma un intervalo de $4^{a}$ aumentada con la nota anterior. Se transcribe del mismo modo. 
Villancico a 4, a la Asunción (1745)

Joseph Conejos Igual (*1709; †1785)

\section{INTRODUCCIÓN}

$1^{\text {a }}$. Al tránsito de María

el cuerpo y alma combaten;

el cuerpo por no dejarla

y el alma por no apartarse. $2^{a}$. No de la unión natural tan estrecho abrazo nace, que vencen los superiores los impulsos naturales.

\section{ESTRIBILLO}

Triunfante señora, ya que tu Asunción se sube de punto quiero alzar la voz.

Manda el rey supremo que porque vivió

María sin culpa, muera sin dolor.
Vivió inmaculada, y así fue razón que muera María conforme vivió.

Merito es su muerte y no obligación, pues pagó el tributo que nunca debió.

\section{RECITADO}

Dime, enigma sagrado, [j]cómo dejas el alto principado que en el nativo suelo gozabas, potestad del mismo cielo[?]
Ya en tu Asunción diviso que aspiras al celeste paraíso, porque el cielo te adora, luz del sol, de la luna y de la aurora.

ARIA
A la que triunfante,
Cantemos la gala
bella emperatriz, diciendo al subir,
huella de los aires, pues vivió sin mancha, la región feliz. que viva sin fin. 
AL TRÁNSITO DE MARÍA (JOSEPH CONEJOS IGUAL, 1745):

UN EJEMPLO DE REUTILIZACIÓN DE TEXTOS EN LOS VILLANCICOS BARROCOS

\subsection{EDICIÓN MUSICAL}

La edición musical se encuentra disponible en este enlace: https://tinyurl.com/y2ftg2rd

\section{BIBLIOGRAFÍA}

Alonso Asenjo, J. (Ed.) (2002-2004). Fiestas por la canonización de Luis Gonzaga y Estanislao de Kostka (Salamanca, 1727). TeatrEsco: Revista del Antiguo Teatro Escolar Hispánico, $\quad \mathrm{n}^{\mathrm{o}} \quad 0 . \quad$ [versión digital]. Recuperado de http://parnaseo.uv.es/Ars/TEATRESCO/documentos/Salamanca\%201727.htm [Acceso: 06.03.2020].

Cruz, sor J. I. de la (1689). Inundación castálida. Madrid: Juan García Infanzón.

Escuer Salcedo, S. (2018). Francisco Viñas y la nueva concepción de la música en la catedral de Jaca (1722-1731). Anuario Musical, LXXIII, pp. 155-168.

Escuer Salcedo, S. (2020). Villancicos y cantadas en la catedral de Jaca (siglo XVIII). Composición, recepción y evolución estilística. Zaragoza: Institución Fernando el Católico.

Escuer Salcedo, S. (2020). Circulación de repertorio en la Corona de Aragón a través del análisis de los fondos del Archivo de Música de la catedral de Jaca. En R. Isusi Fagoaga \& F. Villanueva Serrano (Eds.). La música a la Corona d'Aragó: Investigació, transferència $i$ educació. Valencia: Universitat de València, Institut de Creativitat i Innovacions Educatives [en prensa].

Losada, L. (1727). La juventud triunfante, Representada en las Fiestas, con que celebró el Colegio Real de la Compañia de Jesús de Salamanca la Canonización de San Luis de Gonzaga, y San Stanislao Kostka, y con que aplaudió la Proteccion de las Escuelas Jesuisticas asignada a San Luis Gonzaga por Muestro SS. Padre Benedicto XIII. Salamanca: Eugenio Garcia de Honorato y San Miguel.

Simón Díaz, J. (1982). Bibliografía de la literatura hispánica. Madrid: Consejo Superior de Investigaciones Científicas-Instituto "Miguel de Cervantes" de Filología Hispánica, vol. XII.

Fecha de recepción: 11 de mayo de 2020

Fecha de aceptación: 25 de junio de 2020 